by $10 \%$ each month. The main endpoint was a steroid dependence defined by the recurrence of PMR symptoms and/or the increase of CRP at two times during the decrease of prednisone.

Results: We included 14 patients younger than 60 years old (average age $54+/-$ 0.8 years) and 28 patients older than 65 years old (average age $75.8+/-1.5$ years). The population younger than 60 years was mainly male $(60 \%$ VS $27 \%, p<0.05)$. Both groups were similar in terms of morning stiffness $(2,1 \pm 0,4$ VS $1,9 \pm 0,3$ hours; $p>0.05)$, disease duration ( $4,2 \pm 0,8$ VS $4,1 \pm 0,6$ months; $p>0.05)$, leukocytes rate $(8,3 \pm 1,37$ VS $8 \pm 0,7 \mathrm{G} / \mathrm{L} ; \mathrm{p}>0.05)$ and percentage of antinuclear antibodies rate over $1 / 320(20 \%$ VS $10 \%$; $p>0.05)$. However, regarding to local inflammation, the intensity of FDG uptake highlighted by the Pet scan was lower among young patients (score of $16,9 \pm 1,7$ VS $26,5 \pm 3,0 ; p<0.05$ ). Furthermore, we observed a significant difference concerning therapeutic response according to the age: $60 \%$ of the young patients developed a steroid dependence compared to $20 \%$ in group of old patients $(p<0.05)$. Moreover, the introduction of methotrexate was necessary for $35 \%$ of the young patients against $6.5 \%(p<0.05)$.

Conclusions: Our study is the first to highlight the age as a bad prognosis factor in case of PMR. This difference is independent to the systemic inflammation and surprisingly, local inflammation (assessed by the TEP score) is more important in elderly people. Young patients suffering from PMR are mostly men and are more dependent on steroids. Thus, methotrexate could be straightaway proposed, particularly in patient younger than 60 years old.

Disclosure of Interest: None declared

DOI: 10.1136/annrheumdis-2017-eular.4033

\section{THU0559 PERSISTENT PRURITIC SKIN LESIONS WITH DYSKERATOTIC CELLS IN UPPER LAYER OF EPIDERMIS ARE SPECIFIC AND ASSOCIATED WITH HIGH LEVELS OF SERUM IL-18 IN ADULT-ONSET STILL'S DISEASE}

H. Nishikawa, Y. Taniguchi, N. Aoyama, Y. Terada. Kochi Medical school, Nankoku, Japan

Background: Adult-onset Still's disease (AOSD) is an acute and systemic inflammatory disorder that is characterized by high spiking fever, evanescent rash, arthralgia/arthritis and hyperferritinemia. However, recent reports showed that not only typical evanescent salmon-colored rash but also atypical skin lesions, persistent pruritic papules and plaques, could be associated with AOSD.

Objectives: The aim of this study is to assess the clinical significance of dyskeratotic cells in skin lesions of Japanese patients with AOSD.

Methods: We retrospectively assessed clinical and histological findings of skin lesions including persistent pruritic skin lesions in Japanese patients with AOSD $(n=12)$. Moreover, we compared serological and histological finding of AOSD with that of dermatomyositis (DM) $(n=6)$, drug eruptions (DE) $(n=6)$, and Graft versus Host disease (GVHD) $(n=6)$.

Results: AOSD with persistent pruritic skin lesions $(n=7)$ histologically showed dyskeratotic cells only in upper layer of epidermis and horny layer without intraepidermal infiltrations of inflammatory cells. These dyskeratotic cells were positive by TUNEL and single stranded DNA (sSDNA) stainings, suggesting apoptotic cells. AOSD with evanescent rash $(n=5)$ histologically showed no dyskeratosis. On the other side, the pathological findings of DM $(n=6), D E(n=6)$ and GVHD $(n=6)$ had dyskeratotic cells in all layers of epidermis with inflammatory cells infiltrations. Notably, AOSD with dyskeratosis $(n=7)$ had significant higher levels of serum IL-18 $(74,300 \sim 307,000 \mathrm{pg} / \mathrm{ml})$ than AOSD without dyskeratosis $(\mathrm{n}=5)$.

Conclusions: AOSD with persistent pruritic skin lesions is characterized and specific by prominent epidermal apoptosis, especially involving the upper layers. Therefore, it could play a pivotal role to recognize the atypical skin lesions of AOSD for correct early diagnosis. Finally, the high levels of serum IL-18 might be related with epidermal apoptosis of keratinocyte in AOSD.

Disclosure of Interest: None declared

DOI: 10.1136/annrheumdis-2017-eular.1766

\section{THU0560 DEVELOPMENT OF SYMPTOMS IN VERY EARLY PHASE IN PATIENT WITH ADULT ONSET STILL DISEASE}

H. Tamai, Y. Kaneko, N. Nishina, J. Kikuchi, T. Takeuchi. Division of Rheumatology, Department of Internal Medicine, Keio University School of Medicine, Tokyo, Japan

Background: Adult onset Still Disease (AOSD) is a systemic inflammatory disease presenting various non-specific symptoms like fever, arthralgia and pharyngalgia. Those symptoms are mimicking common cold, which could lead to the delayed diagnosis. However, little is known about the profiles and development of symptoms in very early phase before diagnosis.

Objectives: To clarify the clinical course of AOSD symptom development in very early phase and its effect on the diagnosis delay.

Methods: Consecutive patients with AOSD with enough information in our hospital were enrolled. Initial symptoms before and at diagnosis were investigated in detail. The gradual course from the initial symptoms to the fulfilment of Yamaguchi criteria for AOSD was examined.

Results: A total of 51 patients were enrolled. The mean age at diagnosis was $45.0 \pm 18.9$ years old and $41(80 \%)$ were female. All patients were met with the Yamaguchi criteria. The mean duration from the first symptom to diagnosis was 50.4 days. The duration from the first symptom to the first visit to the medical facility including a general physician was 19.1 days, and that from the first medical facility visit to the first blood test was 8.0 days. While the first symptom was arthralgia in $29(34.1 \%)$, fever in 20 (23.5\%), and eruption in 20 $(23.5 \%)$, pharyngalgia in $13(15.3 \%)$, at diagnosis, fever was found in all patients, eruption in $47(92.2 \%)$, arthralgia in $45(88.2 \%)$, pharyngalgia in $34(66.7 \%)$, lymphadenopathy and/or splenomegaly in 35 (68.6\%), increased white blood cell (WBC) count in 44 (86.3\%), ferritin elevation in 42 (82.4\%), liver enzyme elevation in $41(80.4 \%)$, negative rheumatoid factor (RF) and anti-nucleolar antibody (ANA) in $30(58.8 \%)$. Arthralgia developed 41.9 days prior to the diagnosis, fever 40.9 days, eruption 29.5 days, pharyngalgia 24.5 days and lymphadenopathy and/or splenomegaly 14.8 days. WBC increase was detected 17.8 days prior to the diagnosis, negative RF and ANA 15.1 days, liver enzyme elevation 14.6 days, and ferritin elevation 10.9 days. At 14 days from the first symptom, $32(62.7 \%)$ met 3 of Yamaguchi criteria, 23 (45.1\%) met 4, and 19 (37.3\%) met 5. At 28 days, $40(78.4 \%)$ met 3 of Yamaguchi criteria, $32(62.7 \%)$ met 4, and $27(52.9 \%)$ met 5. The duration to diagnosis was significantly shorter in the patients with eruption developing within 7 days since the first symptoms than those without (35.2 vs 74.3 days, $p=0.03$ )

Conclusions: The mean duration from the first symptom to the diagnosis of AOSD was approximately 50 days, and only a half of the patients met Yamaguchi criteria at 28 days after first symptom. The time of eruption emergence affected the early diagnosis.

Disclosure of Interest: None declared

DOI: 10.1136/annrheumdis-2017-eular.4212

\section{THU0561 CLINICAL PRACTICE GUIDELINE FOR DIAGNOSIS AND MANAGEMENT OF CATASTROPHIC ANTIPHOSPHOLIPID SYNDROME}

K. Legault ${ }^{1}$, C. Hillis ${ }^{1}$, C. Yeung ${ }^{1}$, A. Iorio ${ }^{1}$, M. Crowther ${ }^{1}$, E. Akl ${ }^{2}$, M. Carrier ${ }^{3}$, R. Cervera ${ }^{4}$, F. Dentali $^{5}$, D. Erkan ${ }^{6}$, G. Espinosa ${ }^{7}$, M. Khamashta ${ }^{8}$,

J. Meerpohl ${ }^{9}$, K. Moffat $^{10}$, S. O'Brien ${ }^{11}$, V. Pengo ${ }^{12}$, J. Rand $^{13}$,

I. Rodriguez Pinto ${ }^{7}$, L. Thom ${ }^{14}$, H. Schunemann ${ }^{1} .{ }^{1}$ McMaster University,

Hamilton, Canada; ${ }^{2}$ American University of Beirut, Beirut, Lebanon; ${ }^{3}$ University

of Ottawa, Ottawa, Canada; ${ }^{4}$ University of Barcelona, Barcelona, Spain;

${ }^{5}$ Insubria University, Insubria, Italy; ${ }^{6}$ Weill Medical College of Cornell University,

New York, United States; ${ }^{7}$ Barcelona Hospital Clinic, Barcelona, Spain; ${ }^{8}$ King's

College London, London, United Kingdom; ${ }^{9}$ University of Freiburg, Freiburg,

Germany; ${ }^{10}$ Hamilton Health Sciences, Hamilton, Canada; ${ }^{11}$ Nationwide

Children's, Columbus, United States; ${ }^{12}$ University of Padova, Padua, Italy; ${ }^{13} \mathrm{NY}$

Presbyterian Hospital, New York, United States; ${ }^{14}$ None, Oxford, United Kingdom

Background: Catastrophic antiphospholipid syndrome (CAPS) is characterized by the rapid onset of widespread or multifocal large and/or small vessel thrombosis associated with multi-organ failure in patients meeting the serological criteria for antiphospholipid syndrome [1]. Mortality in CAPS approaches $50 \%$ [2].

Objectives: The RARE-BestPractices project group identified CAPS as a rare disease condition of interest in which to develop a clinical practice guideline. The project was run in partnership with McMaster University, and used the GINMcMaster Guideline Development checklist and Grading of Recommendations Assessment, Development and Evaluation (GRADE) methodology to develop guidelines on rare diseases [3].

Methods: The CAPS guideline was coordinated by a steering committee including representatives from RARE-BP and methodologists from McMaster University. The CAPS guideline panel consisted of 19 international members, including patient representation. The panel used the GradePro software to brainstorm and prioritize potential questions and outcomes. Systematic reviews were performed for each question. To supplement the published evidence, we compiled raw data for mortality from the CAPS Registry, and systematically elicited expert opinion from the panel members using a systematic observation form. For each question an evidence profile and evidence to decision table was generated and shared.

Results: The question prioritization step generated 47 questions, which were ranked to identify the top priorities. The top 10 questions were chosen for guideline development, yielding 7 therapy and 3 diagnostic questions. The outcome generation step yielded 7 outcomes.

The questions were addressed during an in-person panel meeting, held on April 27, 2016 in Barcelona, Spain, with follow-up via webinar on June 3, 2016, and with web-based voting completed July 31, 2016.

Recommendations were developed for all questions and will be discussed in detail.

Conclusions: Ten recommendations were issued by the CAPS Guideline Panel to assist clinicians in diagnosis and management of suspected CAPS patients. Future research is needed to improve evidence quality in rare diseases such as CAPS. The GIN-McMaster Guideline Development Checklist and the GRADE methodology were effective in producing a rigorous guideline in this rare disease. References:

[1] Asherson R. The catastrophic antiphospholipid syndrome. J Rheumatol 1992.

[2] Cervera R, Bucciarelli S, Plasín M, et al. Catastrophic antiphospholipid syndrome (CAPS): descriptive analysis of a series of 280 patients from the "CAPS Registry". J Autoimmun 2009;32:240-5. 